\title{
Three-Dimensional Head-Mounted Display System for Ophthalmic Surgical Procedures
}

$\mathrm{T}$ he term "head-mounted display system" or "helmet-mounted display," both abbreviated HMS, arose from Ivan Sutherland's early experiments with HMSs in the late 1960s. Sutherland constructed an HMS that displayed a simple wireframe model on a binocular display. ${ }^{1}$

The main HMS applications include military, government (fire, police), and civilian-commercial (medicine, video gaming, sports). Military, police, and firefighters use HMSs to display tactical information such as maps or thermal imaging data while viewing a real scene. Recent applications have included the use of HMS for paratroopers.

Live 3D digital imaging has been used during anterior segment ophthalmic procedures since the introduction of the TrueVision 3D surgical system in 2008. The heads-up surgery system in the retina world was introduced by Claus Eckardt in 2014 to overtake several setbacks related to traditional ocular microsurgery using a binocular microscope, mainly in terms of superior ergonomics for the surgeon. ${ }^{2,3}$ In this system, the surgeon performs ophthalmic procedures by viewing the microscopic image on a large flat panel display sent from a 3D camera, by suppressing the use of eyepieces of a microscope.

Herein, the authors describe an innovative technique through the successful use of HMS in the field of ophthalmology, encompassing all the advantages provided by a $3 \mathrm{D}$ display system.

From the *Retina Department, Vitreous Retinal Surgical Unit, Central Lisbon Hospital Center; Lisbon, Portugal; †NOVA Medical School, Universidade NOVA de Lisboa, Lisbon, Portugal; $\ddagger$ Lisbon Retina Institute, Lisbon, Portugal; §Department of Ophthalmology, Portuguese Diabetes Association (APDP), Lisbon, Portugal; qRetina Department, Retinal Surgical Unit, Beatriz Ângelo Hospital; Lisbon, Portugal; **Retina Department, Retinal Surgical Unit, Dr. Gama Pinto Ophthalmology Institute, Lisbon, Portugal; ††Malo Clinic, Lisbon, Portugal; and \$\$ALM, Lisbon, Portugal.

None of the authors has any financial/conflicting interests to disclose.

Supplemental digital content is available for this article. Direct URL citations appear in the printed text and are provided in the HTML and PDF versions of this article on the journal's Web site (www.retinajournal.com).

Reprint requests: Marco Dutra Medeiros, MD, PhD; Lisbon Retina Institute, Duque de Loulé Avenue, nº 5 1050-085, Lisbon, Portugal NOVA Medical School, Universidade NOVA de Lisboa, Lisbon, Portugal e-mail: marcodutramedeiros@gmail.com

\section{Surgical Technique}

The authors describe their first six surgical combined procedures, using the HMS-3000 MT device. All these head-mounted ophthalmic surgical procedures are listed in Table 1. In line, we document and provide our first three surgeries performed under the 3D Side-By-Side Movie system (see Videos, Supplemental Digital Content 1-3, http://links.lww.com/ IAE/A588, http://links.lww.com/IAE/A589, http:// links.lww.com/IAE/A590, which demonstrates the ophthalmic surgical procedures).

All surgeries were performed in the Dr. Gama Pinto Ophthalmology Institute, Lisbon (Figures 1-3). Head-mounted surgeries were performed with a Haag-Streit Surgical microscope HS Hi-R NEO 900 (Haag-Streit Surgical GmbH, Wedel, Germany), and the Sony Head-Mounted System HMS-3000 MT, which provides a 3D color video display of images from the 3D surgical camera systems MCC-3000 MT, the 3D surgical camera systems MCC-3000 MT (2 compact 3D full-HD camera heads that provides exceptional picture quality) (Figure 4). The MCC3000 MT, 2 compact camera heads and one camera control unit, can be easily integrated into a variety of medical systems as well as other compatible 3D medical imaging systems. In addition, a second head-mounted display can be connected to the camera control unit for simultaneous viewing by a second user (Figures 1 and 2).

Depth perception inside an HMS device requires different images for the left and right eyes. There are multiple ways to provide these separate images. The use of dual video inputs offers a completely separate video signal to each eye, which provides the maximum resolution for each image and the maximum frame rate for each eye (see Videos, Supplemental Digital Content 1-3, http://links.lww.com/IAE/A588, http://links. lww.com/IAE/A589, http://links.lww.com/IAE/A590).

\section{Discussion}

The HMS devices are available for use in several fields like entertainment and gaming. The first 
Table 1. List of All Ophthalmic Surgeries Performed Under HMS Device

\begin{tabular}{lll}
\hline Patient & \multicolumn{1}{c}{ Diagnosis } & HMS Procedure \\
\hline 1 & Cataract + ERM & Phacoemulsification/IOL Implantation + PPV + ERM peeling \\
2 & Cataract + macular hole & Phacoemulsification/IOL Implantation + PPV + ILM peeling + 20\% \\
& SF6 Gas \\
3 & Cataract + vitreous hemorrhage & Phacoemulsification/IOL Implantation + PPV + endolaser \\
& photocoagulation \\
4 & Retinal detachment & PPV + silicone oil \\
5 & IOL/capsular bag luxation & PPV/IOL extraction \\
6 & Cataract + Retinal detachment & Phacoemulsification/IOL implantation + PPV 20\% SF6 gas \\
\hline
\end{tabular}

ERM, epiretinal membrane; ILM, internal limiting membrane; IOL, intraocular lens; PPV, pars plana vitrectomy.

commercially available HMS was the Forte VFX1, which was announced at the Consumer Electronics Show in 1994.

Sony, which was another pioneer in this field, introduced the Personal 3D Viewer (or HMZ-T1), a fully surround-sound headset for 3D gaming and movies aimed at the home entertainment market in 2011. With its updating to HMZ-T2 in 2012, Sony ventured into the operating room for its latest headmounted display. This setup allowed surgeons to view high-definition 3D images from inside the patient while performing laparoscopic surgery. The Sony HMS-3000 MT is a personal viewing system that provides a 3D color video display of images from 3D surgical endoscopic/laparoscopic camera systems and other compatible 3D medical imaging systems. This system consists of an HMI-3000 MT image processor unit plus an HMM-3000 MT head-mounted display that provides a stereoscopic visualization from the imaging system.

The display is adjustable for more comfortable wear during extended operating periods, and also allows an unrestricted "look-down" direct viewing while wearing the head-mounted monitor for an unrestricted view of the surgeons hands and the operating area. Unlike passive (circular polarized) 3D systems, the dual-panel design uses two independent OLED panels to display separate images for the wearer's left and right eye. This provides a highresolution $(1,280 \times 720)$ stereoscopic images with precise reproduction of colors and blacks, free of "ghosting" and cross-talk. Because it adds spatial

Fig. 1. Head-mounted surgeries performed by all surgical staff with a Haag-Streit Surgical's microscope HS Hi-R NEO 900 (Haag-Streit Surgical $\mathrm{GmbH}$ ), and the Sony head-mounted system HMS-3000 MT.

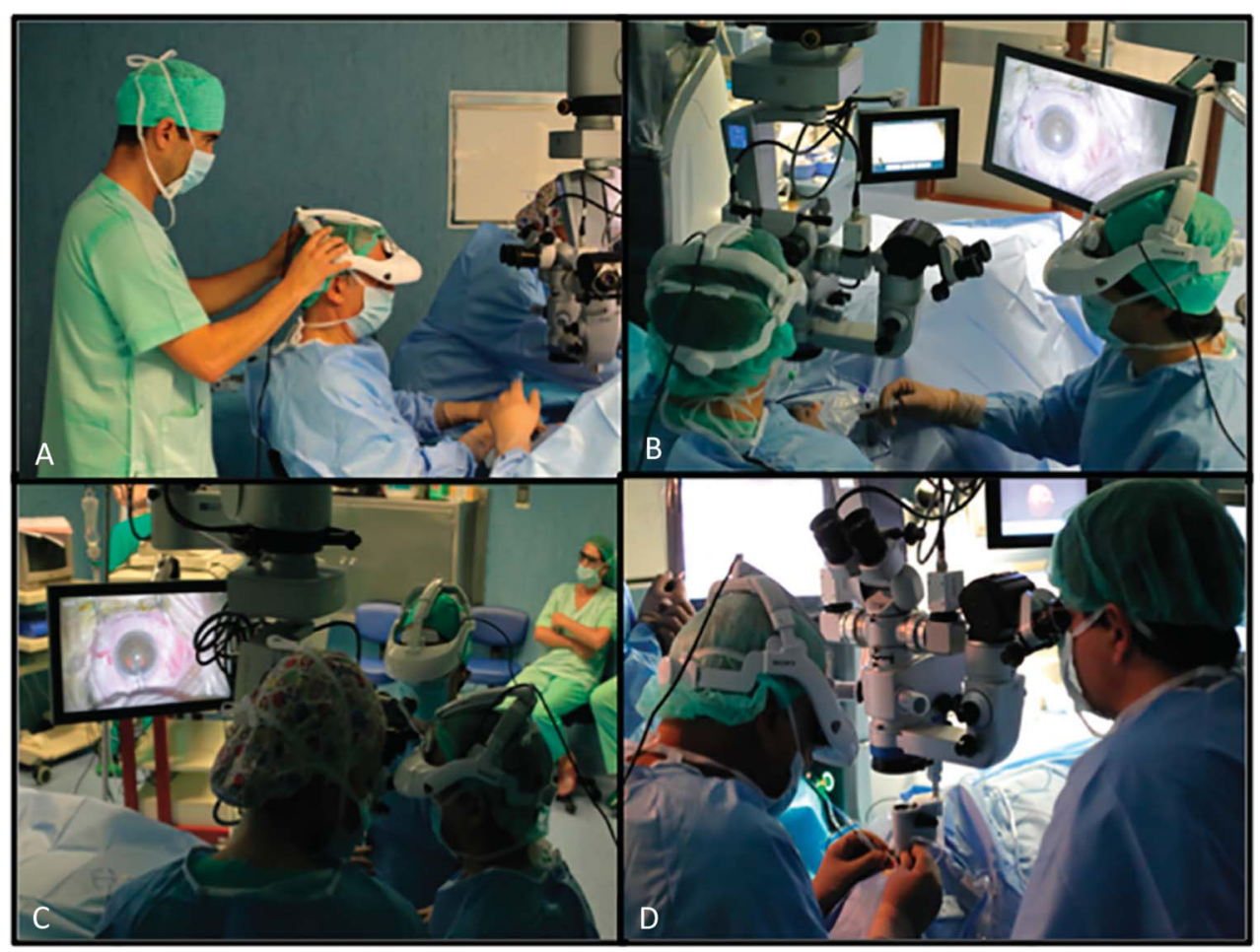




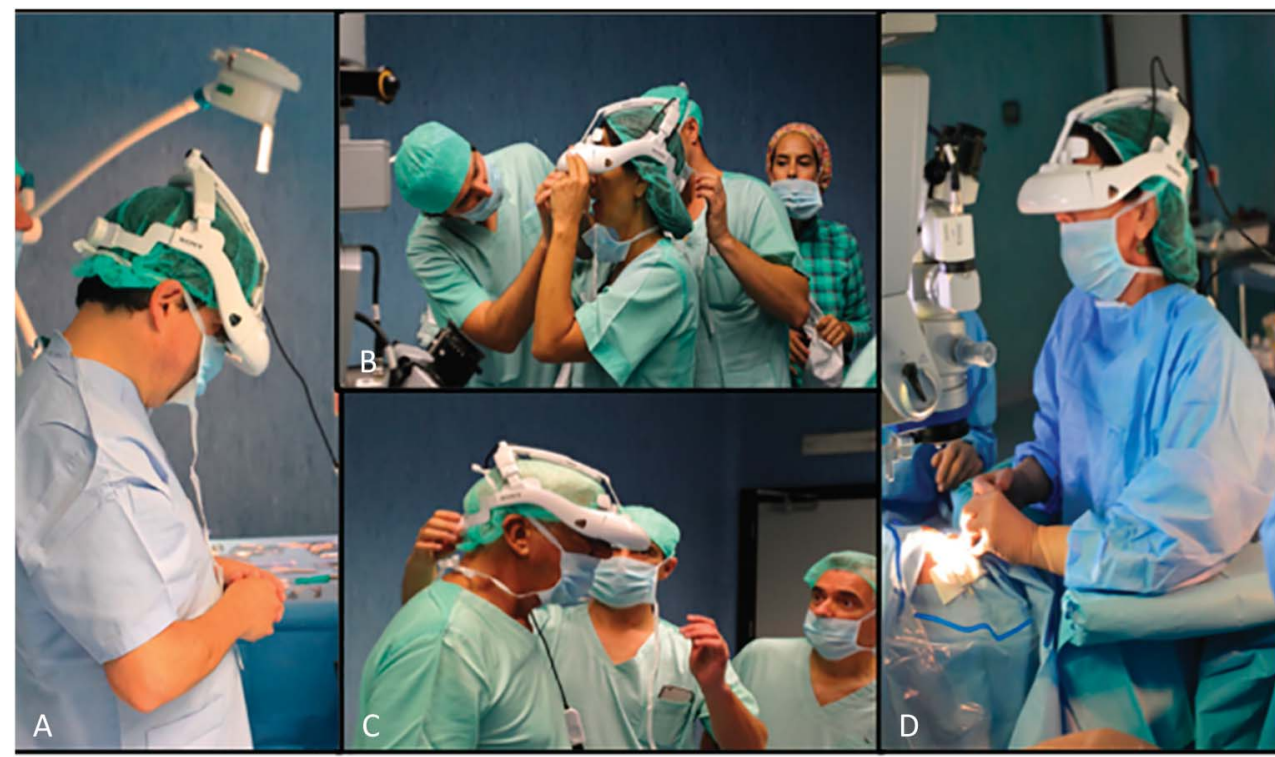

Fig. 2. Head-mounted surgeries performed by all surgical staff with a Haag-Streit Surgical's microscope HS Hi-R NEO 900 (Haag-Streit Surgical $\mathrm{GmbH}$ ), and the Sony head-mounted system HMS-3000 MT.

orientation to medical imaging-and thereby providing a visual experience that is closer to natural sight-3D imaging is fast becoming increasingly popular for medical applications such as microscopic surgery, endoscopic surgery, and roboticassisted surgery, in various surgical specialties. ${ }^{4,5}$ The improved depth perception and spatial orientation provided by 3D can help surgeons to vitrectomize, peel, and cut tissue with safety. A range of image adjustment functions, which includes brightness, contrast, color balance, and sharpness can be adjusted individually, for clearer viewing. Additionally, this system provides a wide $45^{\circ}$ horizontal viewing angle, enabling a more natural visual experience. However, the assistant surgeons also can have the same stereoscopic visualization as the main surgeon, which turns this technology as an excellent tool of live surgery education and training. It should enable an additional tool to Ophthalmologic Training Centers in providing real and simultaneous

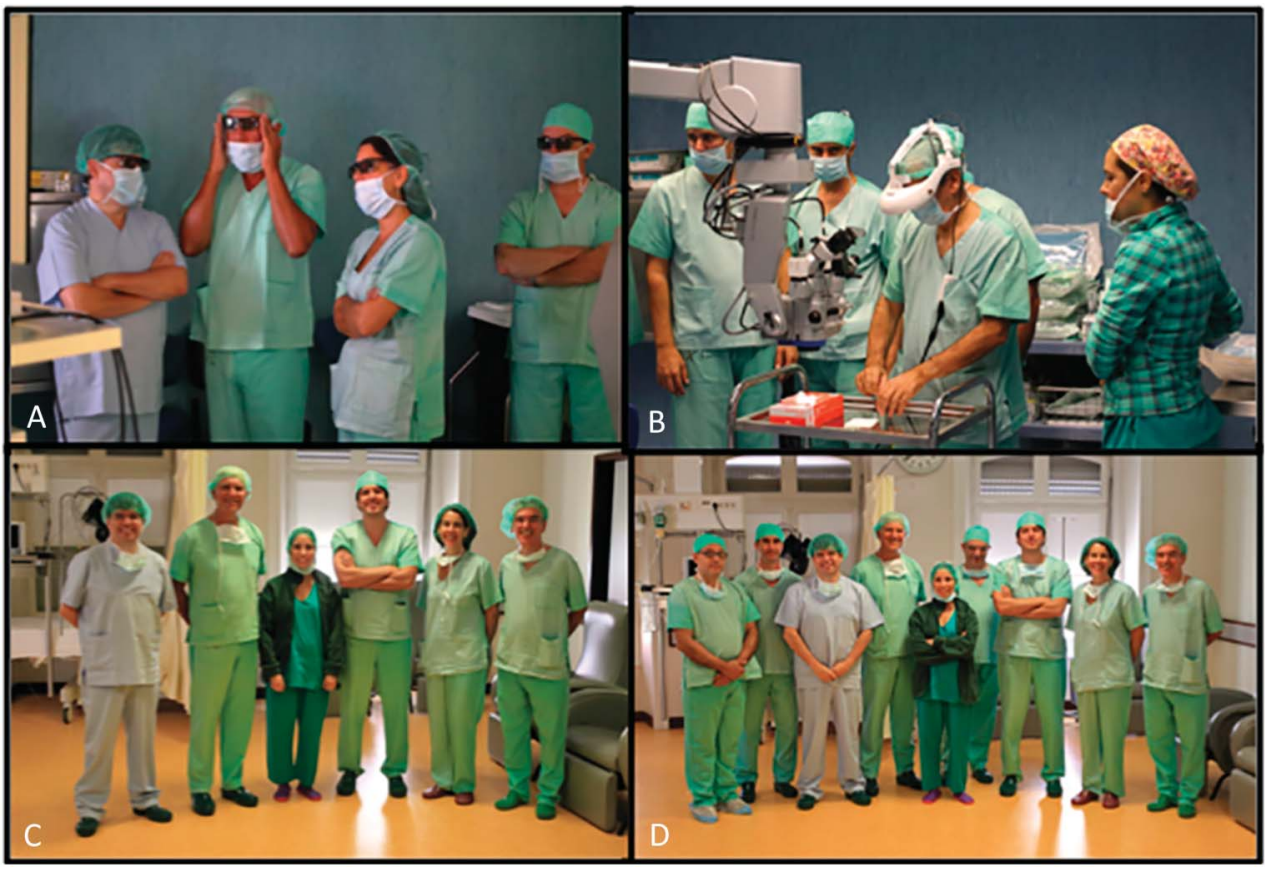

Fig. 3. Surgical staff and Grupo Taper technicians. 
Fig. 4. Haag-Streit Surgical's microscope HS Hi-R NEO 900 (Haag-Streit Surgical GmbH) (part fig. A) and the Sony headmounted system HMS-3000M (part figure B,C), which includes the 3D surgical camera systems MCC-3000 MT (part figure B) (2 compact 3D fullHD camera heads that provides exceptional picture quality).

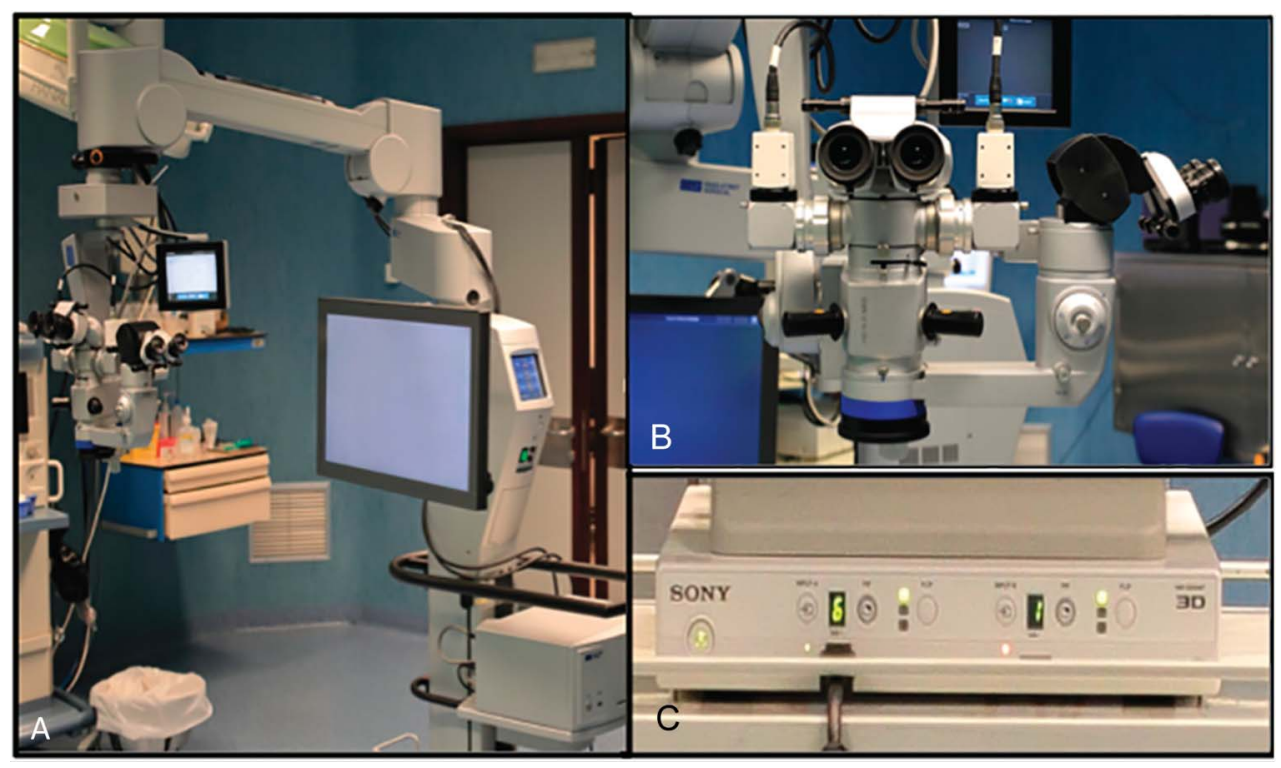

experience to the fellows, depending on the availability of helmets connected to the system. This device is certified for IEC 60601-1 and is in accordance with medical safety standards in the Europe Union, United States, and Canada.

The authors already had some experience with the 3D Heads-Up system. In line, we investigated whether surgical manipulations under HSM conditions would be more difficult compared with traditional microsurgical conditions as well as the Heads-Up system. We also evaluated this new application regarding image resolution and depth of field. In our point of view, a better stereoscopic depth perception was achieved under HSM device.

To the best of our knowledge, this is the first published report addressing the use of "headmounted display system" in the field of ophthalmology. The major limitation of the study is the limited number of patients. We are now working on a larger randomized trial comparing the HMS with the Heads-Up system and the traditional binocular microscope approach. Although the initial results are encouraging and promising, the validation of this technique will be conditional on performing more ophthalmic surgeries.

\section{Ethics Approval}

We have obtained approval by the Gama Pinto Ophthalmology Institute ethics committee.

Key words: 3-dimensional (3D) imaging, headmounted display system, ophthalmic surgical procedures.

\begin{abstract}
MARCO DUTRA-MEDEIROS, MD, PHD*†‡\$ JOÃO NASCIMENTO, MD $\$$ I JOSÉ HENRIQUES, MD***

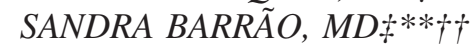
ANA FERNANDES-FONSECA, MD**** NUNO AGUIAR-SILVA, MD* NUNO MOURA-COELHO, MD* VICTOR ÁGOAS, MD**
\end{abstract}

\section{Acknowledgments}

The authors thank GRUPO TAPER PORTUGAL, mainly Engineer Carlos Cunha, Filipe Caninhas, and Armando Leandro for their invaluable collaboration and technical support regarding the Head-Mounted Display System application.

\section{References}

1. Scherffig L. Moving into view: enacting virtual reality. Media Tropes eJournal 2016;1:1913-6005.

2. Eckardt C. Live surgery presentation in 3-D. Frankfurt Retina Meeting; March 15, 2014; Mainz, Germany.

3. Eckardt C, Paulo EB. Heads-up surgery for vitreoretinal procedures: an experimental and clinical study. Retina 2016;36:137-147.

4. How Sony's Head Mount Display System is Taking Endoscopic and Laparoscopic Surgeries Into the Third Dimension. Sony United Kingdom, Inc; 2015. Available at: https://www.sony. co.uk/pro/article/medical-head-mount-display-system-endoscopicand-laparoscopic. Accessed September 23, 2016.

5. Sony Inc. Sapienza University of Rome Holds Study to Evaluate the Sony HMS-3000MT 3DMedical Head Mount Display. Rome, Italy: Institute of Orthopaedic Trauma Surgery. 2014 Available at: https://www. sonypro-latin.com/pro/lang/en/co/ article/medical-sapienzauniversityof- rome-holds-study. Accessed September 24, 2016. 